\title{
Prevalence and factors associated with timely initiation of breastfeeding in Kilimanjaro region, northern Tanzania: a cross-sectional study
}

Frank Kiwango ${ }^{1 *}$, Innocent B. Mboya ${ }^{2,3}$, Beatrice John², Tamara Hashim ${ }^{1,4,5}$, Sia E. Msuya ${ }^{1,2,4}$ and Melina Mgongo ${ }^{1,4,6}$

\begin{abstract}
Background: The World Health Organization (WHO) recommends early initiation of breastfeeding within $1 \mathrm{~h}$ as it confers many benefits to the child and prevents neonatal mortality. This study aimed to determine the prevalence and factors associated with timely initiation of breastfeeding in the Kilimanjaro region, northern Tanzania.

Methods: We analyzed secondary data for 866 participants from a population-based cross-sectional study conducted in April 2016 among mothers with children aged less than 5 years in three districts; Rombo, Same, and Moshi Municipal council in Kilimanjaro region, northern Tanzania. A multistage sampling selected study participants and interviewed using a questionnaire. The generalized linear model, with Poisson family and log-link function was used to estimate the prevalence ratios (PR) and 95\% confidence intervals (Cl) for factors associated with timely initiation of breastfeeding.

Results: The prevalence of timely initiation of breastfeeding was $71.1 \%$. The vast majority of mothers (90.7\%) gave colostrum, and less than a tenth (6.4\%) gave pre-lacteal feed to their children. Adjusted for other factors, not giving children prelacteal feeds remained was significantly associated with a higher prevalence of timely initiation of breastfeeding (PR: $2.22,95 \% \mathrm{Cl} 1.38,3.56, p=0.001$ ). There was no significant association between other characteristics and the likelihood of timely initiation of breastfeeding in this study.

Conclusion: The prevalence of timely initiation of breastfeeding in the Kilimanjaro region was higher than the national estimate. The practice of not giving infants prelacteal feeds increased the likelihood of timely initiation of breastfeeding. There is a need to encourage mothers on the significance of recommended ANC visits and early initiation of breastfeeding to their infants to improve the practice.
\end{abstract}

Keywords: Breastfeeding, Timely initiation, Kilimanjaro, Tanzania

\footnotetext{
* Correspondence: frankkiwango@gmail.com

'Community Health Department, Institute of Public Health, Kilimanjaro

Christian Medical University College (KCMUCo), P. O. Box 2240, Moshi,

Tanzania

Full list of author information is available at the end of the article
}

(c) The Author(s). 2020 Open Access This article is licensed under a Creative Commons Attribution 4.0 International License, which permits use, sharing, adaptation, distribution and reproduction in any medium or format, as long as you give appropriate credit to the original author(s) and the source, provide a link to the Creative Commons licence, and indicate if changes were made. The images or other third party material in this article are included in the article's Creative Commons licence, unless indicated otherwise in a credit line to the material. If material is not included in the article's Creative Commons licence and your intended use is not permitted by statutory regulation or exceeds the permitted use, you will need to obtain permission directly from the copyright holder. To view a copy of this licence, visit http://creativecommons.org/licenses/by/4.0/. The Creative Commons Public Domain Dedication waiver (http://creativecommons.org/publicdomain/zero/1.0/) applies to the data made available in this article, unless otherwise stated in a credit line to the data. 


\section{Background}

Timely initiation of breastfeeding is the provision of a mother's breast milk to infants within $1 \mathrm{~h}$ of birth [1]. The practice of early initiation helps the child get colostrum, which contains antibodies that help protect the infant from common childhood illnesses. Timely/ early initiation of breastfeeding promotes bonding and helps milk production [2]. Literature shows that early initiation of breastfeeding is associated with a $42 \%$ reduction in mortality rate among low birth weight children and a $42 \%$ reduction in infections related to neonatal mortality $[3,4]$ and increases rates of exclusive breastfeeding [5].

The global data shows that coverage of timely initiation of breastfeeding is still below $45 \%$ and declining in several countries. In the developing world, only $44 \%$ of newborns breastfed within $1 \mathrm{~h}$ after delivery [1]. In Latin America and the Caribbean, central and east Europe have lower rates of early initiation of breastfeeding compared to Africa and Asia [4].

There are various factors that influence the timely initiation of breastfeeding. The factors vary from infant to mother's characteristics and health care provider's support and encouragement on the benefits of early initiation of breastfeeding [6-10]. To promote early initiation practices, the WHO has developed different interventions. The interventions include infant and young child feeding strategy, baby-friendly hospital initiative, skin to skin contact right after delivery, the practice of rooming-in, delay cord-cutting, early and continuing mother-infant care [11]. Tanzania has adopted the interventions, but the rate of early initiation of breastfeeding is still low. The Tanzania Demographic Health Survey (TDHS) report shows that only $51 \%$ of infants are breastfed within $1 \mathrm{~h}$ after birth [12].

Some studies have assessed early initiation of breastfeeding at the national level but did not determine the associated factors. Furthermore, existing studies are limited to rural areas such as in the Kilombero and Ulanga districts in the Morogoro region and Rufiji in the Pwani region located in the coastal zone of Tanzania [6]. A previous study in this region reported that $77 \%$ of women practiced early initiation of breastfeeding within $1 \mathrm{~h}$ after delivery [13]. Still, the study did not assess factors related to the practice. Therefore, we aimed to determine prevalence and factors associated with timely initiation of breastfeeding within $1 \mathrm{~h}$ after birth among women with children aged $<24$ months in the Kilimanjaro region, northern Tanzania.

\section{Methods}

\section{Study design and setting}

We performed a secondary analysis of data from a crosssectional study in the Kilimanjaro region, northern Tanzania, conducted by the Institute of Public Health
(IPH) of Kilimanjaro Christian Medical University College (KCMUCo). The study aimed to assess the feeding patterns and nutritional status of children under 5 years of age. Data were collected from three districts of the Kilimanjaro region, namely Same and Rombo district councils and Moshi municipal council. The first two districts are rural while the Moshi municipality being urban, hence allowed for rural-urban comparisons.

\section{Sample size and sampling}

The parent study enrolled 1543 mothers with children aged less than 5 years and was present on the day of data collection. The study used a multistage sampling technique to select study participants. In this analysis, we excluded 541 observations for children aged 24 months or higher and 136 missing information on early initiation of breastfeeding and analyzed data for 866 mothers with children aged 0-24 months (Fig. 1).

\section{Data collection methods}

The institute of public health at KCMUCo collects data during community engagement activities for years 1-3 medical students. The data are open for any student to access when writing their research projects during year 4 of the medical degree training. Trained doctor of medicine students collected data through face to face interviews using a questionnaire (Additional file 1). The questionnaire collected information on the sociodemographic characteristics of mothers, characteristics of children, reproductive history, and breastfeeding (BF) practices. The questionnaire was in both English and Swahili languages but administered using the Swahili language, a language spoken by all the local people in this setting.

\section{Study variables and measurements}

The dependent variable in this study is the early initiation of breastfeeding among mothers with children less than 2 years. Early initiation of breastfeeding was defined as initiating breastfeeding within $1 \mathrm{~h}$ after delivery [1] and assessed by asking mothers, "How long (in hours) after delivery did you start breastfeeding the child?". Mothers who initiated breastfeeding within $1 \mathrm{~h}$ were coded as 1 and 0 if otherwise.

Independent variables included socio-demographic characteristics of mothers, particularly age in years, area of residence, tribe, marital status, education level, and occupation. In contrast, children characteristics included age in months, sex, and birth weight, whereby low birth weight consisted of children born with $<2.5 \mathrm{~kg}$. The reproductive history/ characteristics of the mother included the number of times attended ANC coded as $<4$ visits or $\geq 4$ visits, parity, place of delivery, and whether counseled on breastfeeding during ANC attendance. 


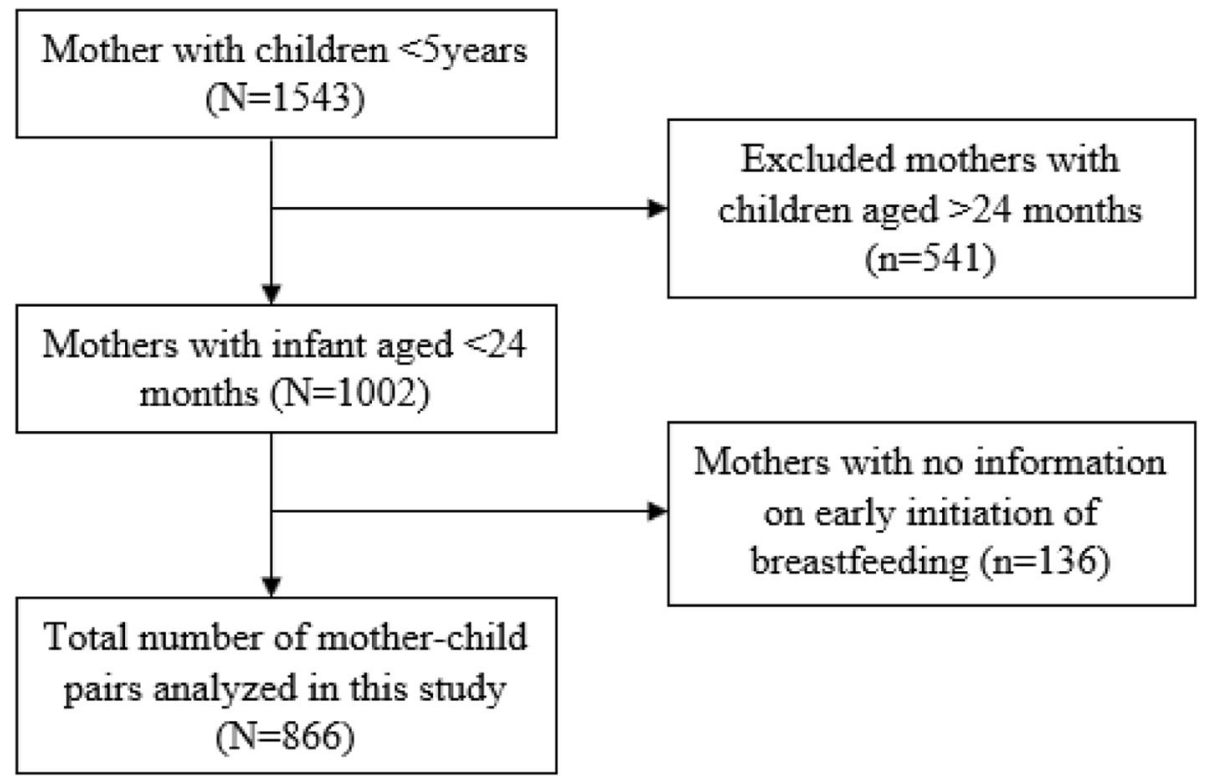

Fig. 1 Flowchart of recruitment and inclusion

Breastfeeding practices included colostrum feeding and prelacteal feeds (defined as giving the child anything apart from breast milk after delivery), coded as Yes or No.

\section{Statistical analysis}

Data were cleaned and analyzed using SPSS version 18 . We summarized categorical variables using frequencies and percentages, and numeric variables using means and standard deviations. The prevalence of timely initiation of breastfeeding was not rare, i.e., greater than $10 \%$. Therefore, we used the generalized linear models, Poisson family and log-link function estimated the prevalence ratios (PR) with their 95\% confidence intervals (CI) for factors associated with timely initiation of breastfeeding instead of classical logistic regression. Variables were significantly associated with timely initiation of breastfeeding if $p$-value $<5 \%$.

\section{Results}

\section{Respondent characteristics}

The mean (SD) age of 866 mothers was 28.3 (SD: 6.8) years, more than two-thirds (36.4\%) were aged 30 years or above, and only $4.8 \%$ were adolescents (15-19 years). The majority $721(83.3 \%)$ were in union (married or cohabiting with their partners), 702 (81.1\%) resided in rural areas, $581(67.1 \%)$ had primary education level, and over half $470(54.3 \%)$ were farmers. Furthermore, the mean (SD) age of children in this study was 12.3 (SD: 7.3) months, and 401 (46.3\%) of all (866) children were aged between 13 and 24 months. About half of them were males 439 (50.7), and 110 (12.7\%) were born with low birth weight (Table 1).

The majority of mothers 767 (88.6\%) delivered in the health facilities, $488(56.4 \%)$ had two children. About two-thirds $548(66.3 \%)$ attended $\geq 4$ ANC visits during the current pregnancy and 284 (32.8\%) of mothers who attended ANC were not counseled on breastfeeding. More than $90 \%$ (90.7\%) of all mothers provided colostrum, while about $6.4 \%$ gave prelacteal feeds to their infants (Table 2). In this study, the prevalence of timely initiation of breastfeeding within $1 \mathrm{~h}$ after birth was 71.1\% (Fig. 2).

\section{Factors associated with timely initiation of breastfeeding}

There were no statistically significant differences in the prevalence of timely initiation of breastfeeding by respondent characteristics, except for prelacteal feeding. In the unadjusted analysis, mothers who did not give their children prelacteal feeding had a higher prevalence of timely initiation of breastfeeding (PR: 2.25, 95\%CI 1.41, $3.60, p=0.001)$ compared to mothers who provided prelacteal feeds. Although adequate $(\geq 4)$ ANC visits during present pregnancy were associated with a higher prevalence of timely initiation of breastfeeding (PR: 1.07, $95 \%$ CI $0.91,1.26)$, this association was not statistically significant (Table 2).

We constructed a multivariable analysis model adjusted for respondent characteristics, including pregnancy history and breastfeeding practices. Adjusted for other factors, not giving children prelacteal feeds remained was significantly associated with a higher prevalence of timely initiation of breastfeeding (PR: 2.22, 
Table 1 Background characteristics of mothers and children $(N=866)$

\begin{tabular}{lll}
\hline Variable & Frequency $(n)$ & Percentage (\%) \\
\hline
\end{tabular}

Socio-demographic characteristics of mothers

Age (years) ${ }^{a}$

$\begin{array}{lll}15-19 & 42 & 4.8 \\ 20-24 & 262 & 30.3 \\ 25-29 & 243 & 28.1 \\ 30+ & 315 & 36.4\end{array}$

Area of residence

Urban

Rural

164

702

Tribe

Chagga

Pare

Others

401

313

152

Marital status

In union

Not in union

Education level

Never been to school 37

Primary education 581

Secondary education and above 248

\section{Occupation}

Farmers

Business

Others

\section{Characteristics of children}

\section{Age (months)}

$$
0-5
$$$$
\text { 6-12 }
$$

$$
\text { 13-24 }
$$

Sex

$$
\text { Male }
$$

Female

\section{Birth weight (Kgs)}

Low birth weight $(<2.5)$

Normal birth weight $(\geq 2.5)$

Reproductive history

\section{Parity}

One child

Two children

$3+$ children

\section{Place of delivery}

Health facility

Home/others
Table 1 Background characteristics of mothers and children $(N=866)$ (Continued)

\begin{tabular}{lll}
\hline Variable & Frequency (n) & Percentage (\%) \\
\hline Number of times attended ANC & & \\
$\quad<4$ visits & 318 & 36.7 \\
$\quad$ 4 visits & 548 & 63.3 \\
Counseled on breastfeeding during ANC visits & \\
$\quad$ Yes & 582 & 67.2 \\
$\quad$ No & 284 & 32.8 \\
Child given colostrum & & \\
Yes & 785 & 90.7 \\
No & 81 & 9.4 \\
Prelacteal feeds & & \\
Yes & 55 & 6.4 \\
No & 811 & 93.7
\end{tabular}

${ }^{\mathrm{a}}$ Frequencies and percentages do not tally to the total due to missing values in mother's age categories

$95 \% \mathrm{CI} 1.38,3.56, p=0.001$ ). Again, mothers with $\geq 4$ ANC visits during present pregnancy were more likely to timely initiate breastfeeding (PR: 1.08 ; 95\% CI 0.91 , 1.27), but this association was not statistically significant. There was no significant association between other characteristics and the likelihood of timely initiation of breastfeeding in this study (Table 2).

\section{Discussion}

The prevalence of early initiation of breastfeeding was $71.1 \%$. The practice of not giving infants prelacteal feeds was the only factor significantly associated with a higher prevalence of timely initiation of breastfeeding in this study.

According to the WHO classification, the estimate reported in this study is categorized as good [14]. These results indicate that a large proportion of infants gets the benefits of early initiation of breastfeeding, which include protection from infections and improving child survival. Early initiation of breastfeeding helps the child get colostrum, which has immune advantages and protect the child from the risk of infections [15]. However, our estimate is slightly lower than (77\%) reported in the previous study done in the Kilimanjaro region [13]. At the national level, only $51 \%$ of mothers initiated breastfeeding within the first hour of life [12]. Though the estimated prevalence was good, about $32 \%$ of women delayed initiating breastfeeding early in this setting. Therefore, there is a need for encouraging mothers to initiate breastfeeding early due to the essential benefits to the child.

The prevalence of early initiation is higher in South Asian countries [4]. Other countries in Europe and Latin America have the lower practice of early initiation of 
Table 2 Factors associated with timely initiation of BF $(N=866)$

\begin{tabular}{|c|c|c|c|c|c|c|}
\hline Variable & $\mathrm{N}$ & TIBF n (\%) & CPR $(95 \% \mathrm{Cl})$ & $\boldsymbol{P}$-value & APR $(95 \% \mathrm{Cl})$ & $\boldsymbol{P}$-value \\
\hline \multicolumn{7}{|c|}{ Socio-demographic characteristics of mothers } \\
\hline \multicolumn{7}{|l|}{ Age (years) } \\
\hline $15-19$ & 42 & $29(69.1)$ & $0.98(0.66,1.44)$ & 0.90 & $1.07(0.70,1.65)$ & 0.75 \\
\hline $20-24$ & 262 & $186(71.0)$ & $1.00(0.83,1.22)$ & 0.98 & $1.08(0.83,1.40)$ & 0.57 \\
\hline $25-29$ & 243 & $175(72.0)$ & $1.02(0.83,1.24)$ & 0.87 & $1.07(0.85,1.34)$ & 0.57 \\
\hline $30+$ & 315 & $223(70.8)$ & 1.00 & & & \\
\hline \multicolumn{7}{|l|}{ Area of residence } \\
\hline Urban & 164 & $116(70.7)$ & $0.99(0.81,1.22)$ & 0.95 & $0.99(0.79,1.24)$ & 0.95 \\
\hline Rural & 702 & $500(71.2)$ & 1.00 & & & \\
\hline \multicolumn{7}{|l|}{ Tribe } \\
\hline Chagga & 401 & $289(72.1)$ & $1.03(0.83,1.29)$ & 0.77 & $1.00(0.79,1.26)$ & 0.98 \\
\hline Pare & 313 & $221(70.6)$ & $1.01(0.80,1.28)$ & 0.92 & $1.00(0.79,1.27)$ & 0.97 \\
\hline Others & 152 & $106(69.7)$ & 1.00 & & & \\
\hline \multicolumn{7}{|l|}{ Marital status } \\
\hline In union & 721 & $523(72.5)$ & $1.13(0.91,1.41)$ & 0.27 & $1.13(0.90,1.43)$ & 0.29 \\
\hline Not in union & 145 & $93(64.1)$ & 1.00 & & & \\
\hline \multicolumn{7}{|l|}{ Education level } \\
\hline Never been to school & 37 & $28(75.7)$ & $1.08(0.73,1.62)$ & 0.69 & $1.04(0.66,1.63)$ & 0.87 \\
\hline Primary education & 581 & $415(71.4)$ & $1.02(0.86,1.22)$ & 0.79 & $0.97(0.80,1.19)$ & 0.80 \\
\hline Secondary education and above & 248 & $173(69.8)$ & 1.00 & & & \\
\hline \multicolumn{7}{|l|}{ Occupation } \\
\hline Farmers & 470 & $342(72.8)$ & $1.07(0.87,1.33)$ & 0.52 & $1.06(0.84,1.32)$ & 0.64 \\
\hline Business & 228 & $160(70.2)$ & $1.03(0.81,1.31)$ & 0.78 & $1.02(0.80,1.31)$ & 0.85 \\
\hline Others & 168 & $114(67.9)$ & 1.00 & & & \\
\hline \multicolumn{7}{|l|}{ Characteristics of children } \\
\hline \multicolumn{7}{|l|}{ Age (months) } \\
\hline $0-5$ & 191 & $137(71.7)$ & $1.01(0.83,1.24)$ & 0.90 & $1.02(0.83,1.26)$ & 0.83 \\
\hline $6-12$ & 274 & $195(71.2)$ & $1.00(0.84,1.21)$ & 0.96 & $1.00(0.84,1.21)$ & 0.96 \\
\hline $13-24$ & 401 & $284(70.8)$ & 1.00 & & & \\
\hline \multicolumn{7}{|l|}{ Sex } \\
\hline Male & 439 & $308(70.2)$ & $0.97(0.83,1.14)$ & 0.73 & $0.97(0.83,1.14)$ & 0.71 \\
\hline Female & 427 & $308(72.1)$ & 1.00 & & & \\
\hline \multicolumn{7}{|l|}{ Birth weight (Kgs) } \\
\hline Low birth weight $(<2.5)$ & 110 & $75(68.2$ & $0.95(0.75,1.21)$ & 0.70 & $0.96(0.75,1.22)$ & 0.72 \\
\hline Normal birth weight $(\geq 2.5)$ & 756 & $541(71.6)$ & 1.00 & & & \\
\hline \multicolumn{7}{|l|}{ Reproductive history } \\
\hline \multicolumn{7}{|l|}{ Parity } \\
\hline One child & 262 & $182(69.5)$ & 1.00 & & & \\
\hline Two children & 488 & $343(70.3)$ & $1.01(0.85,1.21)$ & 0.90 & $1.00(0.80,1.25)$ & 0.995 \\
\hline $3+$ children & 116 & $91(78.5)$ & $1.13(0.88,1.45)$ & 0.34 & $1.15(0.82,1.62)$ & 0.42 \\
\hline \multicolumn{7}{|l|}{ Place of delivery } \\
\hline Health facility & 767 & $553(72.1)$ & $0.88(0.68,1.15)$ & 0.35 & $0.91(0.70,1.19)$ & 0.49 \\
\hline Home/others & 99 & $63(63.6)$ & 1.00 & & & \\
\hline
\end{tabular}

Number of times attended ANC 
Table 2 Factors associated with timely initiation of BF $(N=866)$ (Continued)

\begin{tabular}{|c|c|c|c|c|c|c|}
\hline Variable & $\mathrm{N}$ & TIBF n (\%) & CPR $(95 \% \mathrm{Cl})$ & $\boldsymbol{P}$-value & APR $(95 \% \mathrm{Cl})$ & $\boldsymbol{P}$-value \\
\hline$<4$ visits & 318 & $236(74.2)$ & 1.00 & & & \\
\hline$\geq 4$ visits & 548 & $380(69.3)$ & $1.07(0.91,1.26)$ & 0.41 & $1.08(0.91,1.27)$ & 0.40 \\
\hline \multicolumn{7}{|c|}{ Counseled on breastfeeding during ANC visits } \\
\hline Yes & 582 & $423(72.7)$ & 1.00 & & & \\
\hline No & 284 & $193(68.0)$ & $0.94(0.79,1.11)$ & 0.44 & $0.97(0.81,1.16)$ & 0.74 \\
\hline \multicolumn{7}{|c|}{ Child given colostrum } \\
\hline Yes & 785 & $566(72.1)$ & 1.00 & & & \\
\hline No & 81 & $50(61.7)$ & $0.86(0.64,1.14)$ & 0.29 & $0.88(0.65,1.19)$ & 0.41 \\
\hline \multicolumn{7}{|c|}{ Prelacteal feeds } \\
\hline Yes & 55 & $18(32.7)$ & 1.00 & & & \\
\hline No & 811 & $598(73.7)$ & $2.25(1.41,3.60)$ & 0.001 & $2.22(1.38,3.56)$ & 0.001 \\
\hline
\end{tabular}

TIBF Timely initiated breastfeeding (within $1 \mathrm{~h}$ after birth); CPR Crude odds ratio; APR Adjusted Prevalence Ratio

breastfeeding [4]. In Sub Saharan Africa, Eastern and Southern Africa have better practices of early initiation of breastfeeding, and Ethiopia reported a higher rate (83.1\%) [16].

In this study, $90.6 \%$ of all mothers gave colostrum to their infant, which is a very positive result. Previous studies in Tanzania and other regions showed that mothers were discarding colostrum for the fear that it is dirty or stale milk $[17,18]$. A qualitative study in the Kilimanjaro region reported that mothers give colostrum as they believe it improves child immunity [19]. The finding of the present study indicates that mothers are now aware of the benefits of colostrum feeding.

About $6.4 \%$ of mothers in this study gave prelacteal feeds to their infants. The prelacteal feeds given included infant formula milk, water, glucose, and cow's milk. Not giving infants prelacteal feeds increased the likelihood of timely initiation of breastfeeding in this study. The practice of giving prelacteal feeds is discouraged as it limits the frequency of suckling, and exposes the child to the risk of infections $[1,12,20]$. While ANC attendance during pregnancy was not significantly associated with timely initiation of breastfeeding, there is a need for behavior change interventions and health education, especially during ANC visits to counsel mothers on the risks of giving infants prelacteal. During ANC visits, mothers get the chance to be counseled on proper breastfeeding practices and the benefits to a child's health. These findings are also similar to studies conducted in India and Ethiopia, which showed that ANC attendance was a predictor for timely initiation of breastfeeding $[8,10,21]$.

\section{Strength and limitation and strength of the study}

The strength of this study is that it was community-based and may reflect the early initiation of breastfeeding practice in the Kilimanjaro region. However, this study has some limitations. The prevalence of early initiation of breastfeeding

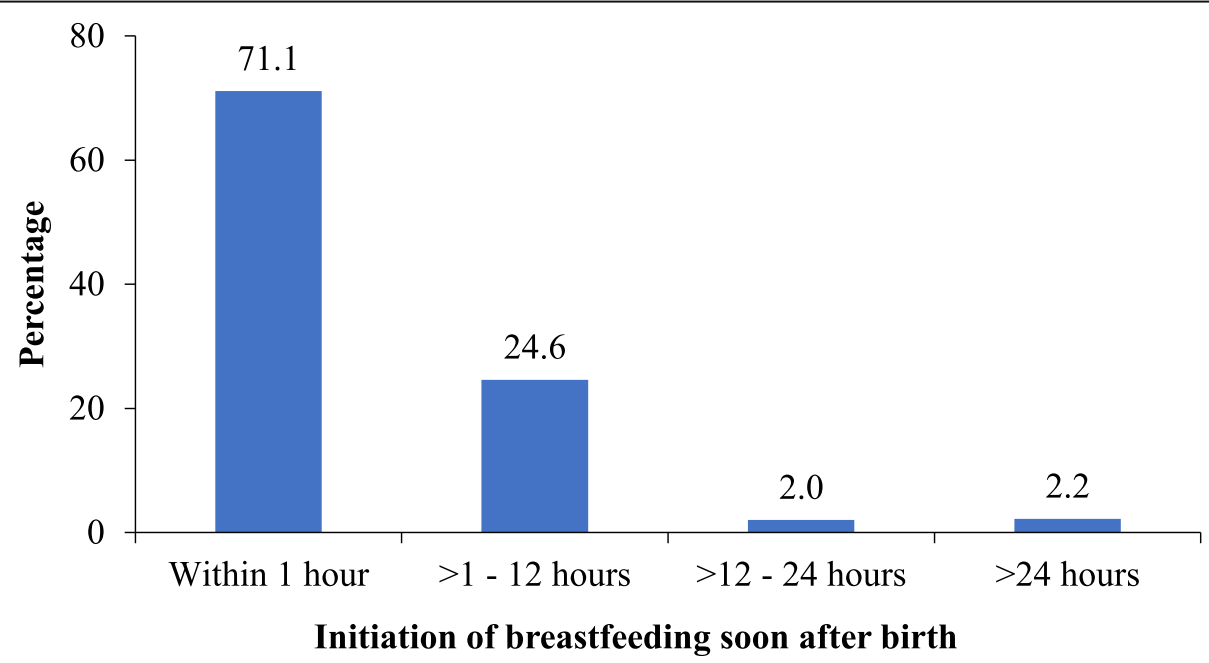

Fig. 2 Percentage distribution of initiation of breastfeeding after delivery 
was based on the mother's ability to remember the practice for the past 2 years. This may lead to recall bias, which is likely to underestimate the prevalence of timely initiation of breastfeeding. Also, our findings may not be generalized to other regions and districts across the country.

\section{Conclusion}

The prevalence of timely initiation of breastfeeding in the Kilimanjaro region was higher than the national estimate. The practice of not giving infants prelacteal feeds increased the likelihood of timely initiation of breastfeeding. There is a need to encourage mothers on the significance of recommended ANC visits and early initiation of breastfeeding to their infants to improve the practice.

\section{Supplementary information}

Supplementary information accompanies this paper at https://doi.org/10. 1186/s12884-020-03209-y.

Additional file 1. Questionnaire

\section{Abbreviations}

ANC: Antenatal Care; AOR: Adjusted Odds Ratio; Cl: Confidence Interval; KCMUCo: Kilimanjaro Christian Medical University College; TDHS: Tanzania Demographic and Health Survey; WHO: World Health Organization

\section{Acknowledgments}

This study was carried as part of doctor of medicine training (for the first author) at Kilimanjaro Christian Medical University College (KCMUCo). We wish to acknowledge all study participants for consenting to take part in this study. The staff of the Institute of Public Health at KCMUCo are also acknowledged for their contribution to this work

\section{Authors' contributions \\ FK: Designing of the study, Wrote the first draft of the manuscript, analysis and interpretation of data. IM: Designing of the study, analysis and interpretation of data. BJ: Designing of the study and analysis of data. SM: Designing of the study and analysis of data. TH: Designing of the study and analysis of data. MM: Designing of the study, analysis and interpretation of data. All authors provided critical review of the manuscript, have read and approved final manuscript for submission.}

\section{Funding}

The only funding for this study was through the government of the United Republic of Tanzania and was assigned for designing, implementation, and local dissemination of the findings.

\section{Availability of data and materials}

The datasets used and/or analyzed during the current study are available from the corresponding author on reasonable request.

\section{Ethics approval and consent to participate}

Ethical approval for the current study was obtained from the Kilimanjaro Christian Medical University College Research and Ethics Review Committee. The permission to use data was obtained from the director, Institute of Public Health of the Kilimanjaro Christian Medical University College.

\section{Consent for publication}

Not applicable.

\section{Competing interests}

All the authors declare that we have no competing interests.

\section{Author details}

${ }^{1}$ Community Health Department, Institute of Public Health, Kilimanjaro Christian Medical University College (KCMUCo), P. O. Box 2240, Moshi,
Tanzania. ${ }^{2}$ Department of Epidemiology and Biostatistics, Institute of Public Health, Kilimanjaro Christian Medical University College (KCMUCo), P. O. Box 2240, Moshi, Tanzania. ${ }^{3}$ School of Mathematics, Statistics \& Computer Science, University of KwaZulu Natal, Pietermaritzburg, Private Bag X01, Scottsville 3209, South Africa. ${ }^{4}$ Better Health for African Mother and Child, P. O. Box 8418, Moshi, Tanzania. ${ }^{5}$ Institute of Basic Medical Sciences, Faculty of Medicine, University of Oslo, Oslo, Norway. ${ }^{6}$ Institute of Clinical Medicine, University of Oslo, Oslo, Norway.

Received: 18 April 2018 Accepted: 25 August 2020

Published online: 01 September 2020

\section{References}

1. Radwan H, Sapsford R. Maternal perceptions and views about breastfeeding practices among Emirati mothers. Food Nutr Bull. 2016;37(1):73-84.

2. Black RE, et al. Maternal and child undernutrition and overweight in lowincome and middle-income countries. Lancet. 2013;382(9890):427-51.

3. Debes AK, et al. Time to initiation of breastfeeding and neonatal mortality and morbidity: a systematic review. BMC Public Health. 2013;13(3):S19.

4. Victora CG, et al. Breastfeeding in the 21st century: epidemiology, mechanisms, and lifelong effect. Lancet. 2016;387(10017):475-90.

5. Fawzi WW. Timing of initiation, patterns of breastfeeding, and infant survival: prospective analysis of pooled data from three randomised trials; 2016.

6. Exavery A, et al. Determinants of early initiation of breastfeeding in rural Tanzania. Int Breastfeed J. 2015;10(1):27.

7. Tewabe T. Timely initiation of breastfeeding and associated factors among mothers in Motta town, east Gojjam zone, Amhara regional state, Ethiopia, 2015: a cross-sectional study. BMC pregnancy and childbirth. 2016;16(1):314.

8. Bimerew A, Teshome M, Kassa GM. Prevalence of timely breastfeeding initiation and associated factors in Dembecha district, north West Ethiopia: a cross-sectional study. Int Breastfeed J. 2016;11(1):28.

9. Berde AS, Yalcin SS. Determinants of early initiation of breastfeeding in Nigeria: a population-based study using the 2013 demograhic and health survey data. BMC Pregnancy Childbirth. 2016;16(1):32

10. Tilahun $\mathrm{G}$, et al. Prevalence and associated factors of timely initiation of breastfeeding among mothers at Debre Berhan town, Ethiopia: a crosssectional study. Int Breastfeed J. 2016;11(1):27.

11. Halpern R, Coelho R. Excessive crying in infants. J Pediatr. 2016;92(3):40-5.

12. Victor $R$, et al. Determinants of breastfeeding indicators among children less than 24 months of age in Tanzania: a secondary analysis of the 2010 Tanzania demographic and health survey. BMJ Open. 2013;3(1):e001529.

13. Mgongo $\mathrm{M}$, et al. Determinants of exclusive breastfeeding in Kilimanjaro region, Tanzania. Science. 2014;2(6):631-5.

14. Organization, W.H., Indicators for assessing infant and young child feeding practices: part 1: definitions: conclusions of a consensus meeting held 6-8 November 2007 in Washington DC, USA. 2008.

15. Friedrich MJ. Early initiation of breastfeeding. JAMA. 2018;320(11):1097.

16. Hailemariam TW, Adeba E, Sufa A. Predictors of early breastfeeding initiation among mothers of children under 24 months of age in rural part of West Ethiopia. BMC Public Health. 2015;15(1):1076.

17. Shirima $\mathrm{R}$, et al. Exclusive breast-feeding is rarely practised in rural and urban Morogoro, Tanzania. Public Health Nutr. 2001;4(2):147-54.

18. Osman H, El Zein L, Wick L. Cultural beliefs that may discourage breastfeeding among Lebanese women: a qualitative analysis. Int Breastfeed J. 2009;4(1):12

19. Mgongo $\mathrm{M}$, et al. "We give water or porridge, but we don't really know what the child wants:" a qualitative study on women's perceptions and practises regarding exclusive breastfeeding in Kilimanjaro region, Tanzania. BMC Pregnancy Childbirth. 2018;18(1):323.

20. Imdad A, Yakoob MY, Bhutta ZA. Effect of breastfeeding promotion interventions on breastfeeding rates, with special focus on developing countries. BMC Public Health. 2011;11(3):S24.

21. Patel A, Banerjee A, Kaletwad A. Factors associated with prelacteal feeding and timely initiation of breastfeeding in hospital-delivered infants in India. J Hum Lact. 2013:29(4):572-8.

\section{Publisher's Note}

Springer Nature remains neutral with regard to jurisdictional claims in published maps and institutional affiliations. 